\title{
Improving Probability Judgment in Intelligence Analysis: From Structured Analysis to Statistical Aggregation
}

\author{
Christopher W. Karvetski ${ }^{*}$, David R. Mandel ${ }^{2 *}$, and Daniel Irwin ${ }^{2}$ \\ ${ }^{1}$ KaDSci LLC \\ ${ }^{2}$ Intelligence, Influence and Collaboration Section, Defence Research and Development Canada \\ *Address correspondence to either David R. Mandel at drmandel66@gmail.com or Christopher \\ W. Karvetski at ckarvetski@gmail.com.
}

\begin{abstract}
As in other areas of expert judgment, intelligence analysis often requires judging the probability that hypotheses are true. Intelligence organizations promote the use of structured methods such as "Analysis of Competing Hypotheses" ( $\mathrm{ACH})$ to improve judgment accuracy and analytic rigor, but these methods have received little empirical testing. In this experiment, we pitted $\mathrm{ACH}$ against a factorized Bayes theorem (FBT) method, and we examined the value of recalibration (coherentization) and aggregation methods for improving the accuracy of probability judgment. Analytic techniques such as ACH and FBT were ineffective in improving accuracy and handling correlated evidence, and $\mathrm{ACH}$ in fact decreased the coherence of probability judgments. In contrast, statistical post-analytic methods (i.e., coherentization and aggregation) yielded large accuracy gains. A wide range of methods for instantiating these techniques were tested. The interactions among the factors considered suggest that prescriptive theorists and interventionists should examine the value of ensembles of judgment-support methods.
\end{abstract}

KEYWORDS: probability judgment; accuracy; Analysis of Competing Hypotheses; Bayes theorem; coherentization; aggregation; intelligence analysis

\section{INTRODUCTION}

In a wide range of professional domains, experts are required to form and update their probabilistic beliefs among competing hypotheses on the basis of imperfect evidence. This is a commonplace task in national security intelligence, and the intelligence community (IC) has adopted the equivalent of judgment-support tools to assist intelligence analysts in this type of assessment. These methods for conducting structured analysis, or "structured analytic techniques" (SATs; CIA, 2009; Heuer \& Pherson, 2014), have been popularized in intelligence training and promoted by upper management, in part as an attempt to avoid past pitfalls (e.g., the 2003 Iraq WMD assessment) and to increase rigor in intelligence analysis (Coulthart, 2016). However, little is known about the effectiveness of such techniques (Chang, Berdini, Mandel, \& Tetlock, 2018; Mandel, 2019; Pool, 2010).

One prominent SAT prescribed to help analysts improve the quality of evidential reasoning and hypothesis testing is the Analysis of Competing Hypotheses ( $\mathrm{ACH})$, formulated 
by CIA analyst Richards Heuer (Heuer, 1999; Heuer \& Pherson, 2014). Core features of ACH include generating a list of mutually exclusive and collectively exhaustive (MECE) hypotheses that represent columns of a spreadsheet with items of evidence listed in rows. Analysts then assess the degree of consistency between each item of evidence and each hypothesis by moving along rows one cell at a time. For each cell they provide a rating from -2 (very inconsistent) to 2 (very consistent). Evidence that is judged not to discriminate between the hypotheses is then removed. Finally, the negative inconsistency scores are summed for each hypothesis (i.e., down each column), and the relative likelihood of the hypotheses is deemed by the rank ordering of the sums. The positive consistency-indicating scores are supposed to be disregarded because Heuer believed that doing so would counteract confirmation bias, which is the search for (or use of) evidence to support a preferred hypothesis (Nickerson, 1998).

Most attempts to document consistency and effectiveness of $\mathrm{ACH}$ have either lacked scientific rigor (for discussion, see Dhami, Belton, \& Mandel, 2019) or arrived at mixed results (Lehner, Adelman, Cheikes, \& Brown, 2008; Folker, 2000). Most insight into the effectiveness of ACH is therefore based on analysts' shared views in survey research (Coulthart, 2016) or critical analyses (e.g., Jones, 2017; Karvetski, Olson, Cross, \& Gantz, 2013a). This has led to calls for increased scientific scrutiny of ACH and SATs, in general (Chang et al., 2018; Dhami, Mandel, Mellers, \& Tetlock, 2015; Mandel \& Tetlock, 2018; National Research Council, 2011; Pool, 2010).

A key criticism is that $\mathrm{ACH}$ is designed for communicating the results of hypothesis tests under conditions of uncertainty, yet $\mathrm{ACH}$ is not a probabilistic technique. An analyst choosing to report probabilities would thus have to do so in an unstructured way that may introduce error or bias. As shown in previous research (Mandel, 2005, 2008; Osherson \& Vardi, 2006; Predd, Osherson, Kulkarni, \& Poor, 2008; Wright, Rowe, Bolger, \& Gammack, 1994), individuals often provide probabilities that violate probability axioms that represent criteria for coherent judgment. For instance, people's probability judgments are often subadditive, adding up to more than is possible (Ayton, 1997; Mandel, 2005, 2008; Rottenstreich \& Tversky, 1997; Tversky \& Koehler, 1994). In a study conducted with intelligence analysts, Mandel, Karvetski, and Dhami (2018) found that intelligence analysts using $\mathrm{ACH}$ showed significantly greater susceptibility to subadditivity than analysts in a control group that did not use any SAT.

In contrast to $\mathrm{ACH}$, Bayesian networks have long been proposed as a form of support for modeling expert judgment within evidential reasoning and hypothesis testing (Fenton, Neil, \& Lagnado, 2013; Kaplan, 1997; Lagnado, Fenton, \& Neil 2013; Ezell, Bennett, von Winterfeldt, Sokolowski, \& Collins 2010; Schum, 1994). As a normative framework for probabilistic belief revision, Bayes' theorem provides a coherent posterior distribution. However, despite the proclaimed benefits and flexibility of Bayesian networks within intelligence analysis (e.g., Karvetski et al., 2013a; Valtorta, Dang, Goradia, Huang, \& Huhns, 2005; Sticha, Buede, \& Rees, 2005; Zlotnick, 1972), there lacks evidence that the application improves judgment accuracy. Due to mathematical complexity and an extensive elicitation process, implementing a Bayesian network typically necessitates the reliance of domain experts (i.e., the intelligence analysts) on a skilled decision consultant/modeler, prompting some skeptics (e.g., Heuer, 2005) to discount the feasibility of analysts using Bayesian networks in their routine work.

Unlike SATs or Bayesian methods, which assist analysts with evidential reasoning prior to judgment, post-analytic statistical methods are mathematical methods that take existing probability judgments as inputs and produce a set of transformed probabilities. Post-analytic statistical methods have proven effective at increasing judgment accuracy in many domains. One 
such method is coherentization, a mathematical recalibration process that remedies incoherence by transforming a set of incoherent probabilities into a coherent set (Karvetski, Olson, Mandel, \& Twardy, 2013b; Osherson \& Vardi, 2006; Predd, Osherson, Kulkarni, \& Poor, 2008) and that guarantees more accurate probability judgments of MECE hypotheses (De Finetti, 1990).

Another post-analytic method popular with risk analysis that has proven useful (although not always, e.g., see MacDonald, Small, \& Morgan 2008) is aggregation, which takes judgments from multiple individuals and derives a single collective judgment (Clemen \& Winkler, 1999; Winkler \& Clemen, 2004). Aggregation can range from two individuals to hundreds or even thousands of individuals ("wisdom of the crowd"; Surowiecki, 2005), and can be combined with simple (equal-weighted) arithmetic averaging or more sophisticated weighting schemes (Hammitt \& Zhang, 2013). While some weighting schemes rely on having seed data with known resolutions to assess the quality of individuals (Cooke, 1990; Cooke \& Goossens, 2008; Van der Fels-Klerx, Cooke, Nauta, Goossens, \& Havelaar, 2005), other methods assign greater weight to more coherent individuals and therefore do not require seed data (Karvetski et al., 2013b; Wang, Kulkarni, Poor, \& Osherson, 2011). Nevertheless, when judged against the equal weighting of individuals, the merit of performance-based weighting schemes is not without question (Bolger \& Rowe, 2014; Cooke, 2015; Fan, Budescu, Mandel, \& Himmelstein, 2019).

\subsection{The Present Research}

The present research aimed to measure the quality of judgment when using $\mathrm{ACH}$, a novel Bayesian elicitation method, coherentization, and aggregation on a classification task that required participants to judge the probabilities of MECE hypotheses using evidential indicators. This research should be of interest to those within the intelligence community that use ACH on a routine basis (analysts), those that rely on the quality of the judgments (policy/decision makers), and those within the risk and decision analysis community that develop, promote, and use Bayesian and post-analytic methods.

The first specific aim of the research was to conceptually replicate key findings in Mandel et al. (2018), which featured an ACH condition and a control condition each consisting of 25 intelligence analysts. As a particular finding in need of replication, Mandel et al. (2018) found that $\mathrm{ACH}$ decreased the analysts' probabilistic coherence and did not improve their accuracy, whereas coherentization and aggregation each significantly increased accuracy. In the present research, we use a different probabilistic hypothesis-testing scenario, recruited a much larger sample size, and elicited a richer set of judgments that permitted both within- and between-subject analyses of each method. We also extended Mandel et al. (2018) by (a) including a Bayesian method to compare with $\mathrm{ACH}$, thus allowing comparison of directly elicited judgments with judgments computed using Bayes' theorem, and (b) investigating the effect of correlated items of evidence.

Whereas Mandel et al. (2018) used a task that assumed a naïve Bayes posterior probability derivation, the present task contained two evidential cues that were perfectly correlated. We manipulated the visual salience of such correlation to test if participants would be more likely to notice the correlation in the high-salience condition and then adjust for the duplicate information using their respective method. We tested two competing hypotheses: on the one hand, participants in the high-salience condition might be more likely to detect the perfect correlation and then discount the contribution of the second, redundant item of evidence. On the other hand, the increased salience might provoke participants to simply (and incorrectly) 
repeat the same judgment for the second item thus leading to double-counting. ACH treats all items of evidence independently regardless of correlation (van Gelder, 2008). Therefore, we did not expect $\mathrm{ACH}$ participants to discount evidence that was statistically redundant and expected to observe many cases of double counting, more so when the salience of the correlation was high than when it was low.

We compared $\mathrm{ACH}$ to a new factorized Bayes' theorem elicitation method (hereafter, FBT) designed to handle correlated evidence. FBT requires the same number of elicitations as $\mathrm{ACH}$ and far less that a full Bayesian network, making it a potentially viable option for routine intelligence analysis and other areas of expert judgment. We hypothesized that FBT, even without training, would sensitize participants to the correlational structure of evidence. Therefore, in contrast to the ACH condition, we did not expect the high-salience condition to promote more double counting of perfectly correlated cues then the low-salience condition.

We further examined the accuracy of the elicited probabilities with and without coherentization (computed probabilities were coherent by design due to Bayes' theorem). Drawing on recent findings (Fan et al., in press; Karvetski et al., 2013b; Mandel et al., 2018; Mellers, Baker, Chen, Mandel, \& Tetlock, 2017; Tsai \& Kirlik, 2012), we hypothesized that coherentization would improve accuracy regardless of whether probabilities were elicited following ACH or FBT methods. We also examined the effect of aggregation on judgment accuracy, studying features such as group size and the type of aggregation method used (e.g., equal weight or coherence-based). A particular interest of ours was to explore the interactions between the analytic and post-analytic methods, given that little research has examined how these methods work if combined.

\section{METHOD}

\subsection{Participants}

One hundred forty-seven participants were recruited using the online crowdsourcing service Qualtrics Panels. Participants were required to be at least 18 years of age, fluent in English, Canadian citizens, and possess at least a Bachelor's degree. Participants were further required to complete the experiment on a computer and smartphones were prohibited. The mean age was 38.21 years $(S D=11.11)$ and $49 \%$ were male.

\subsection{Design and Procedure}

After consenting to participate in the experiment, participants were asked to imagine that they were working on a mushroom classification study conducted on a remote island where they were primarily needed for estimating characteristics based on statistical data (see supplementary materials for a full transcript of task materials). The classifications concerned the toxicity state of mushrooms from the island's population, with toxicity values restricted to three MECE hypotheses: "EDIBLE-NONLETHAL" (EN), "INEDIBLE-NONLETHAL" (IN), or "INEDIBLE-LETHAL" (IL).

Participants were referred to "field-guide" data that represented summary statistics and features of 10,000 randomly-selected mushrooms that were previously classified and grouped into twenty-two species. For each species, the data included the classification label (EN, IN, or IL), the number of species within the sample (and percentage out of 10,000, which ranged from 
$15 \%$ to $0.5 \%$ ), and then binary feature descriptions of cap color (blue or tan), cap shape (bell or convex), gill color (gray or white), and lastly smell, which could take on more than two values (three shown in field guide included earthy, flowery, or fresh). In terms of base rates, the EN, IN, and IL species comprised $25 \%, 35 \%$ and $40 \%$ of the sample, respectively. Participants were told that their task was to use the field-guide data to classify a new mushroom species (Species X).

Participants were randomly assigned to six conditions in a 2 (Method: ACH, FBT) $\times 3$ (Presentation: low-salience, high-salience, conjoined) between-subjects factorial design. Method refers to whether participants were assigned to ACH or FBT conditions. Presentation refers to how two perfectly correlated features were depicted in the field guide, as blue caps were always associated with gray gills, whereas tan caps were always associated with white gills (see supplementary materials for the three displays). The field-guide display was presented throughout the task for participants to reference. In the low-salience condition, the two feature columns were spaced apart to make the correlation less obvious while color coding was used to differentiate the three MECE hypotheses. In the high-salience condition, the feature columns were presented next to each other and the values were color-coded to make the correlation more salient. Finally, in the conjoined condition, the features were presented as in the low-salience condition, but cap color and gill color were combined in a single column as one feature. While the salience of correlation was varied between the high- and low-salience conditions, the conjoined condition eliminated the correlation among the cues. If participants double-counted the correlated items of evidence, this may produce less accurate assessments than in the conjoined condition.

The main task included four steps ${ }^{1}$. First, participants provided probability judgments for a warm-up set of questions. Second, participants provided their initial pre-method probability judgments for a set of questions that comprised the main classification task. Third, participants completed the method (ACH or FBT) as an aid for the main classification task. Finally, participants provided their post-method probability judgments for the same set of questions that comprised the main classification task. The three sets (i.e., warm-up, pre-method, and postmethod) of five questions were worded as follows: "What is the probability that [a randomly selected mushroom, Species X] is [EDIBLE-NONLETHAL, INEDIBLE-NONLETHAL, INEDIBLE-LETHAL, NONLETHAL, INEDIBLE]?" For the warm-up questions (i.e., first set), participants were asked about "a randomly selected mushroom" (e.g., What is the probability that a randomly selected mushroom is EDIBLE-NONLETHAL?). These questions served as a way to benchmark accuracy and coherence when the correct probabilities were obtainable by simply reading values from the field guide and making arithmetic calculations. For the main classification task, both pre-method and post-method, participants were asked about "Species X" (e.g., What is the probability that Species $\mathrm{X}$ is EDIBLE-NONLETHAL?).

Species X was introduced to participants after the warm-up questions as a novel species that had a blue, bell-shaped cap, gray gills, and an acidic smell. The combination of the first three attributes of Species X could be examined in the field guide, but the acidic smell was a novel feature characteristic. Therefore, these questions were expected to be more difficult than the warm-up questions. For each probability elicitation question, participants responded on slider that represented a scale from 0.01 to 0.99 in 0.01 increments. If 0.01 was selected, participants could select $0.01,0.001$ or 0.0001 ; if 0.99 was selected, participants could select $0.99,0.999$ or 0.9999 .

\footnotetext{
${ }^{1}$ After the main task, participants completed questions related to several individual difference measures that were administered for unrelated research purposes.

${ }^{2}$ We did not use the random-response benchmark in tests of our coherence-weighted aggregation methods due to the
} 


\subsubsection{ACH Procedure}

Participants in the ACH condition went through a two-stage process that mimicked ACH as implemented in the PARC tool, which is a software tool that is often used to automate $\mathrm{ACH}$ and was co-developed by Heuer for the US Intelligence Community (PARC, 2006; see supplementary materials for full instructions and visuals of implementation). The ACH matrix was comprised of the three MECE hypotheses at the top with the evidence along the left column consisting of the four indicator values ordered for the appropriate condition (three indicator values in the conjoined presentation condition, which combined cap color and gill color), plus the base rates of the different types ( $25 \%$ of mushrooms in field guide were EN, $35 \%$ were IN, and $40 \%$ were IL). The data was described as completely trustworthy, and thus only (in)consistency inputs were used (i.e., ACH ratings did not include reliability/credibility). In brief, participants were required (a) to populate the matrix cells with ratings of how consistent each item of evidence was with each hypothesis, which ranged from very inconsistent $(-2)$, inconsistent (-1), neutral/not applicable (0), consistent (1), or very consistent (2), (b) to revise the matrix by eliminating rows that they judged to be nondiagnostic, and (c) to calculate inconsistency scores for the three hypotheses by summing the negative score values. Participants could view their completed ACH matrices, along with the field-guide data, while responding to the five post-method probability questions about Species X.

\subsubsection{FBT Procedure}

The general FBT model used within the experiment is obtained by first designating one random variable as the set of $k$ MECE hypotheses, $\mathrm{H}=\left\{\mathrm{H}_{1}, \ldots, \mathrm{H}_{\mathrm{k}}\right\}$. Letting $\mathrm{q}_{\mathrm{i}}$ represent an observed state of indicator random variable $\mathrm{Q}_{\mathrm{i}}, Q=\left\{\mathrm{q}_{1}, \ldots, \mathrm{q}_{\mathrm{m}}\right\}$ can be defined as a set of $m$ observed indicators. For any $H_{p} \in \mathrm{H}$ we have the general form Bayes' theorem as follows:

or equivalently:

$$
P\left(H_{p} \mid Q\right)=\frac{P\left(Q \mid H_{p}\right) P\left(H_{p}\right)}{P(Q)}
$$

$$
P\left(H_{p} \mid q_{1}, \ldots, q_{m}\right)=\frac{P\left(q_{1}, \ldots, q_{m} \mid H_{p}\right) P\left(H_{p}\right)}{P\left(q_{1}, \ldots, q_{m}\right)}
$$

Expanding the denominator, equation (2) can be re-written as:

$$
P\left(H_{p} \mid q_{1}, \ldots, q_{m}\right)=\frac{P\left(q_{1}, \ldots, q_{m} \mid H_{p}\right) P\left(H_{p}\right)}{\sum_{i=1}^{k} P\left(q_{1}, \ldots, q_{m} \mid H_{i}\right) P\left(H_{i}\right)} .
$$

FBT is obtained by applying the chain rule for conditional probabilities whereby each $P\left(q_{1}, \ldots, q_{m} \mid H_{i}\right)$ term is re-written as:

$$
P\left(q_{1}, \ldots, q_{m} \mid H_{i}\right)=P\left(q_{m} \mid q_{m-1}, \ldots, q_{1}, H_{i}\right) \times P\left(q_{m-1} \mid q_{m-2}, \ldots, q_{1}, H_{i}\right) \times \cdots \times P\left(q_{1} \mid H_{i}\right)
$$

yielding an assumption-free decomposition of Bayes' theorem that enables consideration of each new indicator in light of each hypothesis, as well as any previously assessed indicators.

Recalling that Species X had a blue, bell-shaped cap, gray gills, and acidic smell (abbreviated "B," "BS," "G," and "A," respectively), a task-specific representation of FBT for the EN hypothesis in the low-salience presentation is as follows:

with

$$
P(E N \mid B, B S, G, A)=\frac{P(B, B S, G, A \mid E N) P(E N)}{P(B, B S, G, A \mid E N) P(E N)+P(B, B S, G, A \mid I N) P(I N)+P(B, B S, G, A \mid I L) P(I L)}
$$




$$
\begin{aligned}
& P(B, B S, G, A \mid E N) P(E N)= \\
& P(A \mid B, B S, G, E N) P(G \mid B, B S, E N) P(B S \mid B, E N) P(B \mid E N) P(E N), \\
& P(B, B S, G, A \mid I N) P(I N) \\
& \quad=P(A \mid B, B S, G, I N) P(G \mid B, B S, I N) P(B S \mid B, I N) P(B \mid I N) P(I N),
\end{aligned}
$$

and

$$
\begin{aligned}
& P(B, B S, G, A \mid I L) P(I L)= \\
& P(A \mid B, B S, G, I L) P(G \mid B, B S, I L) P(B S \mid B, I L) P(B \mid I L) P(I L) .
\end{aligned}
$$

Participants in the FBT condition were sequentially presented with three sets of questions corresponding to the presentation-specific formulas presented in equations (6) to (8) (see supplementary materials for exact questions). After providing judgments for the FBT inputs, participants also directly provided a set of probability judgments for the five questions related to the classification task (i.e., FBT direct), which were compared with the probabilities calculated using the FBT inputs (i.e., FBT computed).

\subsection{Coherentization}

The first three probability judgments (EN, IN, IL) in the aforementioned question sets (i.e., warm-up, pre-method, and post-method) are subject to the unitarity constraint and their total probability should equal 1 . The remaining two probability judgments referred to implicit disjunctions and are subject to the additivity constraint: $\mathrm{P}(\mathrm{NONLETHAL})=\mathrm{P}(\mathrm{EN})+\mathrm{P}(\mathrm{IN})$ and $\mathrm{P}(\mathrm{INEDIBLE})=\mathrm{P}(\mathrm{IN})+\mathrm{P}(\mathrm{IL})$. We examined two forms of coherentization: a unitarity-only form (U-form) that applied only the unitarity constraint and a unitarity-and-additivity form (UAform) that applied both constraints. For example, if the raw probability judgments provided by participant $j$ for the five questions are denoted as $y_{j}^{R}=\left(y_{1, j}^{R}, y_{2, j}^{R}, y_{3, j}^{R}, y_{4, j}^{R}, y_{5, j}^{R}\right)=(0.74,0.18$, $0.26,0.75,0.38$ ) for $\mathrm{P}(\mathrm{EN}), \mathrm{P}(\mathrm{IN}), \mathrm{P}(\mathrm{IL}), \mathrm{P}(\mathrm{NONLETHAL})$, and $\mathrm{P}(\mathrm{INEDIBLE})$, respectively, these values violate both the unitarity and additivity constraints. U-form coherentization yields $y_{j}^{C}=(0.68,0.12,0.20)$ for $\mathrm{P}(\mathrm{EN}), \mathrm{P}(\mathrm{IN}), \mathrm{P}(\mathrm{IL})$, whereas UA-form coherentization yields judgments $y_{j}^{C}=(0.65,0.12,0.23,0.77,0.35)$ for the five values. For U-form coherentization, we can define a non-negative incoherence metric as:

$$
I M_{j}^{U}=\sqrt{\sum_{i=1}^{3}\left(y_{i j}^{R}-y_{i j}^{C}\right)^{2}},
$$

and similarly for UA-form coherentization as:

$$
I M_{j}^{U A}=\sqrt{\sum_{i=1}^{5}\left(y_{i j}^{R}-y_{i j}^{C}\right)^{2}} .
$$

In the example above, we have with $I M_{j}^{U}=0.10$ and $I M_{j}^{U A}=0.12$. Also, since the probabilities for the three MECE hypotheses sum to more than 1, we can describe this as subaddtivity or a positive bias from unitarity of 0.18 , which is calculated as:

$$
\operatorname{Bias}_{j}^{U}=\left[\sum_{i=1}^{3} y_{i j}^{R}\right]-1 \text {. }
$$

Analogously, we can define two measures of bias from additivity for the NONLETHAL union:

and for the INEDIBLE union:

$$
\text { Bias }_{j}^{N}=\mathrm{y}_{1 j}+\mathrm{y}_{2 j}-\mathrm{y}_{4 j}
$$




$$
\operatorname{Bias}_{j}^{I}=\mathrm{y}_{2 j}+\mathrm{y}_{3 j}-\mathrm{y}_{5 j} .
$$

In our example, $\operatorname{Bias}_{j}^{N}=0.17$ and $\operatorname{Bias}_{j}^{I}=0.06$.

\subsection{Aggregation}

We implemented equal-weighted averaging of both the raw judgments $y_{j}^{R}$ and the coherentized judgments $y_{j}^{C}$. Given $n$ participants (where $2 \leq n \leq N$ ) the equal-weighted raw aggregate probability judgment of the $i^{\text {th }}$ hypothesis $(\mathrm{i}=1,2,3)$ is defined as:

$$
\bar{y}_{i}^{R}=\frac{1}{n} \sum_{j=1}^{n} y_{i j}^{R},
$$

whereas the equal-weighted coherentized aggregate probability judgment is defined as:

$$
\bar{y}_{i}^{C}=\frac{1}{n} \sum_{j=1}^{n} y_{i j}^{C} .
$$

Since the coherentized judgments are a convex set, the aggregate judgment $\bar{y}^{C}$, being a linear combination of elements from the set, is also coherent.

Implementing coherence-based weighting required converting the incoherence metrics to weight values using a weighting function (similar to that of Wang et al., 2011)

$$
\omega_{j}=e^{\left(-I M_{j} \times \beta\right)},
$$

yielding the coherence-weighted, coherentized aggregate probability judgment as:

$$
\bar{y}_{i}^{C, W}=\sum_{j=1}^{n} \omega_{j} y_{i j}^{C} / \sum_{j=1}^{n} \omega_{j} .
$$

The weighting function in equation (16) assigns a weight value of 1 to the $j$ th individual if $\mathrm{IM}_{j}=$ 0 or if $\beta=0$. When $\beta=0,(15)$ and equation (17) are equivalent, but when $\beta>0$, incoherent participants are increasingly penalized. While there is some guidance as to how to set an optimal $\beta$ parameter in part to minimize the fifty-fifty blip (Karvetski et al, 2013b; Bruine de Bruin, Fischbeck, Stiber, \& Fischhoff, 1999; Fischhoff \& Bruine de Bruin, 1999), we varied $\beta$ in the results to test sensitivity.

\subsection{Measuring Accuracy}

Provided with a truth vector $x=(\mathrm{P}(\mathrm{EN}), \mathrm{P}(\mathrm{IN}), \mathrm{P}(\mathrm{IL}))=\left(\mathrm{x}_{1}, \mathrm{x}_{2}, \mathrm{x}_{3}\right)$ the measure of accuracy we used in the experiment is mean absolute error (MAE):

$$
M A E=\frac{1}{3 n} \sum_{i=1}^{3} \sum_{j=1}^{n}\left|y_{i j}-x_{i}\right| .
$$

The summation across $i$ again refers to the set of three hypotheses (and excludes the event unions of NONLETHAL and INEDIBLE). While mean-square-error (or Brier) scores are generally preferred for probabilistic forecasting as they are proper rules (Brier, 1950; Gneiting \& Raftery, 2007), and could have been used interchangeably as a metric of accuracy, participants were not being rewarded based on accuracy thus properness was not an important feature. Additionally, MAE has the favorable scoring characteristic of not being overly sensitive to outliers (Willmott \& Matsuura, 2005).

When investigating the accuracy of individuals $\left(y_{i j}=y_{i j}^{R}\right.$ or $\left.y_{i j}^{C}\right), n$ refers to the number of individuals in the considered group. For aggregation $\left(y_{i j}=\bar{y}_{i}^{R}, \bar{y}_{i}^{C}\right.$, or $\left.\bar{y}_{i}^{C, W}\right), n$ refers to the number of simulations run at each group size. For the warm-up questions, the truth vector concerning a randomly-selected mushroom was the base rate among the three types: $.25, .35$, and 
.40 for EN, IN, and IL, respectively.

For Species X, the combination of the first three features could be found within the EN, IN, and IL types in the field guide and the FBT decomposition could be used to determine the initial posterior probabilities that comprised the information of the first three features. However, obtaining the final truth vector that accounted for all features required updating the initial posterior probabilities using likelihood ratios of the novel acidic smell (i.e., $\mathrm{P}(\mathrm{A} \mid \mathrm{B}, \mathrm{BS}, \mathrm{G}, \mathrm{EN}) /$ $\mathrm{P}(\mathrm{A} \mid \mathrm{B}, \mathrm{BS}, \mathrm{G}, \mathrm{IL}), \mathrm{P}(\mathrm{A} \mid \mathrm{B}, \mathrm{BS}, \mathrm{G}, \mathrm{EN}) / \mathrm{P}(\mathrm{A} \mid \mathrm{B}, \mathrm{BS}, \mathrm{G}, \mathrm{IN})$, and $\mathrm{P}(\mathrm{A} \mid \mathrm{B}, \mathrm{BS}, \mathrm{G}, \mathrm{IN}) / \mathrm{P}(\mathrm{A} \mid \mathrm{B}$, $\mathrm{BS}, \mathrm{G}, \mathrm{IL}))$, which were incalculable from the field-guide data and therefore required a direct elicitation. To avoid potentially biasing participants, we recruited a separate sample of 53 participants meeting the same inclusion criteria as the original participants. These participants provided the values needed to derive the average likelihood ratios of acidic feature (see supplementary materials for details), which were used to calculate a final truth vector for Species $\mathrm{X}$, yielding posterior probabilities of .44 for $\mathrm{EN}, .21$ for IN, and .35 for IL.

\section{RESULTS}

\subsection{Assessment of Correlated Cues}

We examined how participants assessed the two perfectly correlated features in the $\mathrm{ACH}$ and FBT conditions as a function of whether they were assigned to the low-salience or highsalience condition. In the ACH condition, participants' consistency ratings were coded as (a) rational if, for the subsequently presented feature (gill color), participants moved non-zero entries of cap color to zero or reduced the magnitude of nonzero entries of cap color; (b) doublecounting if the entire row for the cap color was the same for gill color; and (c) other-irrational for any other response, such as increasing the magnitude of an input or switching the sign. Table I shows the proportion of participants exhibiting each response type with Clopper-Pearson 95\% confidence intervals in brackets. Results for tests of the difference in proportions between the low- and high-salience conditions are shown in the last three columns. Supporting the hypothesis that salient redundancies in evidence trigger double counting, a significantly greater proportion of double counting was evident in the high-salience condition than in the low-salience condition when participants used $\mathrm{ACH}$.

In the FBT condition, we examined the treatment of correlated cues using the mean absolute distance (MAD) between the conditional probabilities provided by participants assigned to these two correlated features. If participants failed to condition properly, but rather entered the same conditional probabilities for gill color as they did with cap color, expected MAD would be zero. Consistent with double-counting, MAD was significantly lower in the high-salience condition $(M$ $=0.14, S D=0.19)$ than in the low-salience condition $(M=0.27, S D=0.25), t(53.7)=2.15, p=$ .036 , Cohen's $d=0.56$.

\subsection{Validity Check}

As a validity check on the difficulty of main classification task, we tested whether the (easier) warm-up set of judgments were more coherent and accurate than the (harder) pre-method judgments of classifying Species X. As Table II shows, warm-up judgments were significantly more accurate and more coherent than the pre-method judgments. These findings provide a successful validity check of participants' effort and the difficulty level of the tasks. 


\subsection{Individuals' Judgment Coherence}

To examine coherence within the main classification task (i.e., Species X), we first analyzed participants' incoherence metrics derived from their direct pre-method judgments as well as direct post-method judgments. We excluded the FBT-computed probabilities because, as noted earlier, these are coherent by design. Both $\mathrm{IM}^{\mathrm{U}}$ and $\mathrm{IM}^{\mathrm{UA}}$ were analyzed in a 3 $($ Presentation $) \times 2($ Method $) \times 2$ (Timing) mixed analysis of variance $\left(\right.$ ANOVA). For both $\mathrm{IM}^{\mathrm{U}}$ and $\mathrm{IM}^{\mathrm{UA}}$, none of the main or interaction effects approached significance, $p \mathrm{~s}>.07$. Therefore, there were comparable degrees of incoherence across experimental conditions and elicitation times.

Whereas the IM scores captured systematic and nonsystematic components of incoherence, we conducted two sets of additional tests that examined systematic forms of incoherence. Our first set of analyses examined bias from unitarity, Bias ${ }_{j}^{U}$. As before, we conducted a three-way mixed ANOVA but substituted bias from unitarity as the dependent variable. This analysis revealed a significant main effect of timing, $F(1,141)=12.29, p=.0006$. This effect was qualified by a significant method $\times$ timing interaction effect, $F(1,141)=22.12, p$ $<.0001$. No other effect in the model was significant, $p \mathrm{~s}>.19$. Table III shows the average bias from unitarity and statistical tests for both $\mathrm{ACH}$ and FBT direct estimates. The increase in bias in the $\mathrm{ACH}$ condition represents a large effect, and is consistent with the subadditivity effects predicted by support theory (Tversky \& Koehler, 1994). The same bias is notably absent in the FBT condition.

Our second test of systematic incoherence examined bias from additivity using both Bias $_{j}^{N}$ and Bias $s_{j}^{I}$. We subjected the two bias metrics to a four-way (Presentation x Method x Timing $\mathrm{x}$ Bias type) mixed ANOVA, where the bias type referred to either Bias ${ }_{j}^{N}$ or Bias $_{j}^{I}$. Once again, we found a significant effect of timing, $F(1,141)=6.64, p=.01$. This effect was qualified by a significant method $\times$ timing interaction effect, $F(1,141)=4.18, p=.04$. No other effects in the model were significant, $p \mathrm{~s}>.20$. Table IV shows the average bias from additivity and statistical tests for both ACH and FBT. Consistent with our tests of unitarity bias, ACH significantly increased additivity bias, whereas FBT did not.

\subsection{Individuals' Accuracy}

We examined the accuracy of participants' direct and computed post-method probability judgments. Using a 3 (Presentation) $\times 3$ (Method: ACH, FBT direct, FBT computed) ANOVA, we found no significant main or interaction effect on MAE ( $p \mathrm{~s}>.28)$. Next, we examined the accuracy of direct judgments (i.e., excluding the FBT computed judgments) in a 3 (Presentation) $\times 2$ (Method) $\times 2$ (Timing) x 3 (Coherentization: raw, U-form, UA-form) mixed ANOVA. There was a significant main effect of coherentization, $F(2,140)=151.80, p<.0001$. This effect was qualified by a coherentization $\times$ timing interaction effect, $F(2,140)=4.44, p=.01$. No other effect in the model was significant, $p \mathrm{~s}>.23$. As Table $\mathrm{V}$ shows, both forms of coherentization improved accuracy compared to the raw judgments. The average standardized effect comparing raw judgments to coherentized variants across timeframes was large, average $d=1.10$. The two forms of coherentization did not differ in the pre-method judgments, although there was a significant improvement in accuracy for the UA-form compared to the U-form in the post- 
method judgments.

\subsection{Aggregation}

Building on the preceding results, we investigated the accuracy of aggregated probability judgments. We generated 1,000 bootstrap samples of participants at group sizes ranging from 1 to 49 by increments of 2 and then applied multiple aggregation techniques. To benchmark participants' performance, we also aggregated random responses, where judgments of $\mathrm{P}(\mathrm{IN})$, $\mathrm{P}(\mathrm{EN}), \mathrm{P}(\mathrm{IN}), \mathrm{P}(\mathrm{NONLETHAL})$, and $\mathrm{P}(\mathrm{INEDIBLE})$ were drawn from a $\mathrm{U}(0,1)$ distribution.

Figure 1 displays MAE with unweighed aggregation over various methods, timings, and coherentization schemes. Focusing on the raw estimates (left column of Figure 1), the analyses confirmed that participants were more accurate than the random-response data, yet the shape of the curves (including points of diminishing returns) for ACH and FBT direct estimates were similar to that of the random data. Since the random response data had no signal, aside from the initial gap near the group size equal to 1, the improvement of aggregation for the ACH and FBT direct estimates were likely due to reduction in variance. Among raw probability judgments, the FBT computed values produced the most accurate estimates, having the steepest initial improvement with continuing improvement as a function of group size. Also, it is evident that the $\mathrm{ACH}$ aggregate raw judgments were less accurate post-method than pre-method.

The U-form probability judgments (middle column of Figure 1) showed substantial improvement for ACH and FBT direct aggregate judgments, yet we found similar expected gains with the random-response data, and the FBT-computed probability judgments were most accurate for group sizes greater than 7. Lastly, the UA-form judgments (third column) produced the most accurate post-method judgments, but this finding should be interpreted cautiously as the improvement was also observed for the random-response data, and thus was likely attributable to our specific truth vector. Note that with UA-form coherentization, post-method judgment accuracy was the best in the ACH condition over all group sizes.

Figure 2 shows the signed Cohen's $d$ effect size for selected pariwise comparisons as a function of group size. The decreasing slope in the two top-row panels shows that application of the ACH and FBT methods to raw judgments tends to make them worse, and this method decrement increases with group size and more so for the $\mathrm{ACH}$ method. The positive slope in the bottom-row panels shows the accuracy advantage of the FBT-computed method over no method (i.e., FBT pre-method on the bottom left) or the FBT-direct method (bottom right).

Figure 3 shows the signed Cohen's $d$ effect size for pairwise comparisons of raw, Uform, and UA-form aggregated post-method judgments by group size and method. Randomresponse data (top row) were included as a benchmark. For example, in the top-left panel, the Uform coherentization of random data provided a large-effect increase in accuracy over the raw values for a group size of 1 , and that this effect increased in magnitude over larger group sizes. The remaining panels in the first column shows the effect sizes for U-form versus raw judgments using the post-method ACH direct and the FBT direct judgments. The middle column shows similar effects for the UA-form versus raw judgments, while the right column shows the comparions between the U-form and UA-form judgments (a positive effect implies UA was more accurate). It is evident from the first two columns of Figure 3 that the improvement in accuracy due to coherentization and agregation show a similar trajectory over group size for human judgments and for the random-response data. Only in comparisons of the two methods do we observe noticably steeper positive curves for the human judgments than in the random- 
response data, which indicate an accuracy advantage for UA-form coherentization over U-form coherentization that increases with group size.

Whereas all preceding analyses used equal weighting $(\beta=0)$, the next set of analyses examined the sensitivity of the results where individuals were weighted by coherence. In particular, we aimed to investigate accuracy sensitivity for different methods of measuring coherence and incorporating coherence-weighting in aggregation (cf. Fan et al., 2019). Figure 4 shows MAE by group size when coherence weighting of $U$-form post-method direct judgments is applied within the ACH and FBT groups for a) different scale values of $\beta$ (different panels) and with $b$ ) weights derived from different estimates and/or coherence schemes (different line colors). For example, the "Post-method Species X, U-weighted" curve signifies that the weights were derived from equation (17) using the post-method $\mathrm{IM}^{\mathrm{U}}$ values (i.e., task-specific), whereas the "Pre-method Random, UA-weighted" curve implies the weights were derived from the the $\mathrm{IM}^{\mathrm{UA}}$ values associated with the warm-up questions. Finally, the black curve in each panel represents the equal weighting of U-form coherentized post-method judgments. Overall, equalweighting yielded the best accuracy for smaller group sizes, and was near the middle of the range of accuracies for larger group sizes. However, the rank ordering of weighting types was dependent on method. The most accurate weighting type in the $\mathrm{ACH}$ group at larger group sizes (weights derived from the post-method U-form coherentization) was the least accurate type for the FBT group. Akin to Figure 4, Figure 5 shows aggregation results using the post-method UAform judgments for weighting (as opposed to U-form). Once again, equal-weighting was the most robust aggregation technique, especially for smaller group sizes. Note further that the postmethod UA-form weighting, which was the task-specific scheme, was one of least accurate methods at larger group sizes for $\mathrm{ACH}$.

A final set of analyses examined the benefits of averaging input judgments for FBT and then computing a single posterior set of judgments versus averaging the FBT-computed judgments. Since we had both the inputs values as well as the computed posterior values for the FBT group, our aim was to test if averaging the inputs to Bayes theorem produced more accurate results than averaging the outputs of Bayes theorem. In other words, if each expert is represented by a Bayesian model (e.g., Stiber, Small, \& Pantazidou, 2004), can we determine the best way to aggregate? Figure 6 shows MAE for these averaging schemes over group size and presentation. We included the latter factor because each condition resulted in different FBT questions due to feature ordering. Overall, aggregating the computed posterior probabilities provided the most accurate estimates in the low-salience and conjoined condition, whereas the two approaches (i.e., averaging FBT inputs vs. averaging FBT outputs) were comparable in the high-salience condition.

\section{DISCUSSION}

The primary aim of this research was to examine alternative methods for improving the accuracy of probability judgments rendered in the context of a hypothesis-testing task. The task used was designed to include some evidential sources that were correlated, as would be expected to occur in real intelligence problems. For instance, an analyst might receive similar information from two informants who coordinated their messages in advance, perhaps in an attempt to make the information appear more reliable or influential. The task we used also required participants to integrate statistical information that was relevant to their hypothesis testing with subjective judgments regarding novel sources of information that could not be directly estimated from 
statistical sources (namely, a cue value for a target species that had never been reported before).

The experiment yielded several important findings. One key set of findings concerns the effectiveness of the analytic methods evaluated in improving the accuracy and coherence of probability judgments. Participants instructed to use ACH were significantly less coherent (in terms of both unitarity and additivity violations) and also less accurate after using the technique than they were prior to using it. These findings add to a body of evidence suggesting that $\mathrm{ACH}$ does not improve judgment quality and may in fact impede some aspects of quality (e.g., Dhami et al., 2019; Mandel et al., 2018; Whitesmith, 2019). The FBT method produced better results than $\mathrm{ACH}$, but they were nevertheless lacklustre. Participants were no more or less coherent after using FBT than they were before using it, but their accuracy was impaired by its use when assessed on the basis of their direct posterior probability judgments. In short, simply following the elicitation steps in the FBT method did not help participants accurately judge on their own the posterior probabilities of the hypotheses they were evaluating. Nor did the FBT method as a basis for computing posterior probabilities from their other FBT judgments show promise when used without aggregation. However, when (unweighted) aggregation was employed, the FBT computation method showed a distinct accuracy advantage over direct judgment methods. Therefore, aggregation of FBT-computed judgments shows promise and represents a simpler alternative to aggregating results from multiple experts' Bayes nets.

Another key set of findings of our research is that post-analytic methods yielded large improvements in accuracy. Both forms of coherentization that we tested improved accuracy, and the average improvement corresponded to just over a 1 SD reduction in error. Likewise, all forms of aggregation substantially improved accuracy. The steepest gains were achieved over relatively small group sizes, with most of the gains realized with a group size of 10 . This is consistent with other studies showing that most of the benefit of aggregation can be achieved with relatively small statistical groups (Mandel et al., 2018, Winkler \& Clemen, 2004). By including a random-response benchmark in our analyses, we are also able to show that much of the observed benefit of aggregation is not due to wisdom-of-the-crowd effects (Surowiecki, 2005) but rather reduction in judgment variance (i.e., the effect of Jensen's [1906] inequality). Accuracy curves that show steeper and more sustained gains than random-response data might signal processes that go beyond the effects of variance reduction. In our research, such characteristics are most clearly associated with FBT-computed aggregate judgments in tests involving equal weighting, which shows both greater steepness and is more sustained than the accuracy curves for the alternative methods. ${ }^{2}$ It would be instructive if in future research, dataanalytic methods for parsing accuracy gains into meaningful sources (e.g., variance reduction, crowd wisdom) were developed.

A third important set of findings from this research concerns the interactions between alternative methods for improving judgment accuracy. We explored competing analytic methods, including $\mathrm{ACH}$ and FBT, and competing post-analytic methods, including coherentization using different constraints as well as equal-weighted aggregation versus a variety of coherenceweighted aggregation. Consistent with Fan et al. (2019), our findings indicate that researchers and practitioners alike who seek to improve judgment accuracy should consider the performance of ensembles of methods. Consider ACH: on its own it was the least effective method, actually reducing accuracy and coherence compared to a within-subject no-method control. However, when $\mathrm{ACH}$ was paired with UA-form coherentization and unweighted aggregation with group

\footnotetext{
${ }^{2}$ We did not use the random-response benchmark in tests of our coherence-weighted aggregation methods due to the already large number of accuracy curves resulting from the variables considered.
} 
sizes of five or more, it was effective at minimizing error. Yet even a "slight" change from UAform coherentization to U-form coherentization was sufficient to change that result. Similarly, it is evident from our findings that the effect of the aggregation weighting method is also sensitive to the many factors (group size, method used to derive inputs, weighting derivation, etc.), and the ensemble method that was most effective with one set of factors might be least effective with a slight modification (e.g., as observed in Figure 4).

Given the importance of taking an ensemble approach to judgment support, what best practices are suggested by our findings? First, the findings unequivocally support recalibrating forecasts so that they are coherent. In this research, using multiple constraints-namely, unitarity and additivity — paid off (see also Karvetski et al., 2013b). However, other studies have found better results when only the unitarity constraint is applied (Fan et al., 2019; Mandel et al., 2018). Although we cannot explain this variability at this time, it is clear that the largest effect is between any form of coherentization and none. Second, the findings show that aggregation benefits accuracy, and that, consistent with earlier research (Ashton \& Ashton, 1985; Libby \& Blashfield, 1978; Winkler \& Clemen, 2004), substantial gains can be achieved with small group sizes. Third, the effects of coherentization and aggregation are additive and both should be employed when feasible. Fourth, unless there are opportunities for running validation tests, it may be prudent to forego coherence weighting and adopt a simpler unweighted aggregation scheme. Fifth, our findings indicate that where aggregation of Bayesian estimates is performed, it is more effective to average the posterior probability estimates of individuals than to average their priors and likelihoods first and then compute a posterior probability distribution using those aggregated inputs.

Finally, given that we did not provide training to participants on either analytic method, future research could investigate the use of training on both methods to examine whether it might reduce the observed errors and biases. For example, training within the FBT condition might include direction on how to form conditional probabilities and could provide feedback on the accuracy of practice judgments. We expect differential effects of training with ACH and FBT. In Mandel et al. (2018), the ACH group included analysts who were recently trained in ACH, yet use of ACH made analysts' judgment less coherent and did not benefit accuracy, just as we observed in the present experiment. In contrast, Mandel (2015) and Tetlock and Gardner (2015) found that even brief training in probability theory can be effective at improving probabilistic judgment skill. We observed that participants in both conditions were prone to double counting perfectly correlated evidence even when the relationship was made highly salient. A key difference between the conditions, however, is that with $\mathrm{ACH}$, providing the same inputs is not necessarily wrong, as the method offers no guidance on how to deal with correlated evidence. In contrast, with FBT, participants who ignore the correlational structure of information are not formulating the correct conditional probabilities required for FBT. Future research should therefore explore the value of training to improve the accuracy payoff of the factorized Bayes theorem method developed here.

\section{ACKNOWLEDGEMENTS}

This research was funded by Canadian Safety and Security Program project CSSP-TI2016-2224. We thank Norman Fenton and David Lagnado for feedback on our methods, two anonymous reviewers for feedback on earlier drafts of this paper, and Brenda Fraser and Tonya 
Hendriks for their research support.

\section{REFERENCES}

Ashton, A. H., \& Ashton, R. H. (1985). Aggregating subjective forecasts: Some empirical results. Management Science, 31(12), 1499-1508.

Ayton, P. (1997). How to be incoherent and seductive: Bookmakers' odds and support theory. Organizational Behavior and Human Decision Processes, 72(1), 99-115.

Barnes, A. (2016). Making intelligence analysis more intelligent: Using numeric probabilities. Intelligence and National Security, 31(3), 327-344.

Bolger, F., Rowe, G. (2014). The aggregation of expert judgment: do good things come to those who weight? Risk Analysis, 35(1), 5-11.

Brier, G. W. 1950. Verification of forecasts expressed in terms of probability. Monthly Weather Review, 78(1), 1-3.

Bruine de Bruin, W., Fischbeck, P. S., Stiber, N. A., \& Fischhoff, B. (2002). What number is "fifty-fifty"?: Redistributing excessive $50 \%$ responses in elicited probabilities. Risk Analysis, 22(4), 713-723.

Chang, W., Berdini, E., Mandel, D. R., \& Tetlock, P. E. (2018). Restructuring structured analytic techniques in intelligence. Intelligence and National Security, 33(3), 337-356.

Central Intelligence Agency. (2009). A tradecraft primer: Structured analytic techniques for improving intelligence analysis. Accessed April 4, 2019:

https://www.cia.gov/library/center-for-the-study-of-intelligence/csi-publications/booksand-monographs/Tradecraft\%20Primer-apr09.pdf

Clemen, R. T., \& Winkler, R. L. (1999). Combining probability distributions from experts in risk analysis. Risk Analysis, 19(2), 187-203.

Cooke, R. M. (1990). Statistics in expert resolution: A theory of weights for combining expert opinion. In R. Cooke \& D. Costantini (Eds.), Statistics in science (pp. 41-72). Dordrecht, The Netherlands: Springer.

Cooke, R.M. (2015). The aggregation of expert judgment: do good things come to those who weight? Risk Analysis, 35(1), 12-15.

Cooke, R.M., \& Goossens, L.L. (2008). TU Delft expert judgment data base. Reliability Engineering \& System Safety, 93(5), 657-674.

Coulthart, S. (2016). Why do analysts use structured analytic techniques? an in-depth study of an American intelligence agency. Intelligence and National Security, 31(7), 933-948.

De Finetti, B. (1990). Theory of probability: A critical introductory treatment. New York, NY: John Wiley and Sons.

Dhami, M. K., Mandel, D. R., Mellers, B. A., \& Tetlock, P. E. (2015). Improving intelligence analysis with decision science. Perspectives on Psychological Science, 10(6), 753-757.

Dhami, M. K., Belton, I. B., \& Mandel, D. R. (2019). The 'Analysis of Competing Hypotheses' in intelligence analysis. Applied Cognitive Psychology, 33(6), 1080-1090.

Ezell, B. C., Bennett, S. P., von Winterfeldt, D., Sokolowski, J., \& Collins, A. J. (2010). Probabilistic risk analysis and terrorism risk. Risk Analysis, 30(4), 575-589.

Fan, Y., Budescu, D. V., Mandel, D. R., \& Himmelstein, M. (2019). Improving accuracy by coherence weighting of direct and ratio probability judgments. Decision Analysis, 16, 197-217.

Fenton, N., Neil, M., \& Lagnado, D. A. (2013). A general structure for legal arguments about 
evidence using bayesian networks. Cognitive Science, 37(1), 61-102.

Fischhoff, B., \& Bruine de Bruin, W. J. A. (1999). Fifty-fifty=50\%? Journal of Behavioral Decision Making, 12(2), 149-163.

Folker, R. D. (2000). Intelligence analysis in theater joint intelligence centers: An experiment in applying structured methods. Accessed February 19 ${ }^{\text {th }}, 2018$ at: https://fas.org/irp/eprint/folker.pdf

Gneiting, T. and Raftery, A.E. (2007). Strictly proper scoring rules, prediction, and estimation. Journal of the American Statistical Association, 102(477), 359-378.

Hammitt, J.K., \& Zhang, Y. (2013). Combining Experts' Judgments: Comparison of Algorithmic Methods Using Synthetic Data. Risk Analysis, 33(1), 109-120.

Heuer, R. J., \& Center for the Study of Intelligence (U.S.). (1999). Psychology of intelligence analysis. Washington, DC: Center for the Study of Intelligence, Central Intelligence Agency.

Heuer, R. J. (2005). How does Analysis of Competing Hypotheses (ACH) improve intelligence analysis? Accessed February 19 ${ }^{\text {th }}, 2018$ at: http://www.pherson.org/wpcontent/uploads/2013/06/06.-How-Does-ACH-Improve-Analysis_FINAL.pdf

Heuer, R. J., Jr., \& Pherson, R.H. (2014). Structured analytic techniques for intelligence analysis. Washington, DC: CQ Press.

Jensen, J. L. W. V. (1906). Sur les fonctions convexes et les inégalités entre les valeurs moyennes. Acta Mathematica, 30(1), 175-193.

Jones, N. (2018). Critical epistemology for analysis of competing hypotheses. Intelligence and National Security, 33(2), 273-289.

Kaplan, S. (1997). The words of risk analysis. Risk Analysis, 17(4), 407-417.

Karvetski, C. W., Olson, K. C., Gantz, D. T., \& Cross, G. A. (2013a). Structuring and analyzing competing hypotheses with Bayesian networks for intelligence analysis. EURO Journal on Decision Processes, 1(3), 205-231.

Karvetski, C. W., Olson, K. C., Mandel, D. R., \& Twardy, C. R. (2013b). Probabilistic coherence weighting for optimizing expert forecasts. Decision Analysis, 10(4), 305-326.

Lagnado, D., Fenton, N., \& Neil, M. (2013). Legal idioms: A framework for evidential reasoning. Argument and Computation, 4(1), 46-63.

Lehner, P. E., Adelman, L., Cheikes, B. A., \& Brown, M. J. (2008). Confirmation bias in complex analyses. IEEE Transactions on Systems, Man, and Cybernetics - Part A: Systems and Humans, 38(3), 584-592.

Libby, R., \& Blashfield, R. K. (1978). Performance of a composite as a function of the number of judges. Organizational Behavior and Human Performance, 21(2), 121-129.

MacDonald, J.A., Small, M.J. \& Morgan, M.G. (2008). Explosion probability of unexploded ordnance: Expert beliefs. Risk Analysis, 28(4), 825-841.

Mandel, D. R. (2005). Are risk assessments of a terrorist attack coherent? Journal of Experimental Psychology: Applied, 11(4), 277-288.

Mandel, D. R. (2008). Violations of coherence in subjective probability: A representational and assessment processes account. Cognition, 106(1), 130-156.

Mandel, D. R. (2015). Instruction in information structuring improves bayesian judgment in intelligence analysts. Frontiers in Psychology, 6, 387. doi:10.3389/fpsyg.2015.00387

Mandel, D. R. (2019). Can decision science improve intelligence analysis? In S. Coulthart, M. Landon-Murray, \& D. Van Puyvelde (Eds), Researching national security intelligence: Multidisciplinary perspectives (pp. 117-140). Washington, DC: Georgetown University 
Press.

Mandel, D. R., \& Barnes, A. (2018). Geopolitical forecasting skill in strategic intelligence. Journal of Behavioral Decision Making, 31(1), 127-137.

Mandel, D. R., Karvetski, C. W., \& Dhami, M. K. (2018). Boosting intelligence analysts' judgment accuracy: What works, what fails? Judgment and Decision Making, 13(6), 607621.

Mandel, D. R., \& Tetlock, P. E. (2018). Correcting judgment correctives in national security intelligence. Frontiers in Psychology, 9, 2640.

Mellers, B. A., Baker, J. D., Chen, E., Mandel, D. R., \& Tetlock, P. E. (2017). How generalizable is good judgment? A multi-task, multi-benchmark study. Judgment and Decision Making, 12(4), 369-381.

National Research Council (U.S.). (2011). Intelligence analysis for tomorrow: Advances from the behavioral and social sciences. Washington, DC: National Academies Press.

Nelson, J. D. (2005). Finding useful questions: On Bayesian diagnosticity, probability, impact, and information gain. Psychological Review, 112(4), 979-999.

Nickerson, R. S. (1998). Confirmation bias: A ubiquitous phenomenon in many guises. Review of General Psychology, 2(2), 175-220.

Osherson, D., \& Vardi, M. Y. (2006). Aggregating disparate estimates of chance. Games and Economic Behavior, 56(1), 148-173.

Palo Alto Research Center (PARC). (2006). ACH: Version 2.0.3.

Pool, R. (2010). Field evaluation in the intelligence and counterintelligence context: Workshop summary. Washington, DC: National Academies Press.

Predd, J. B., Osherson, D. N., Kulkarni, S. R., \& Poor, H. V. (2008). Aggregating probabilistic forecasts from incoherent and abstaining experts. Decision Analysis, 5(4), 177-189.

Rottenstreich, Y., \& Tversky, A. (1997). Unpacking, repacking, and anchoring: Advances in support theory. Psychological Review, 104(2), 406-415.

Schum, D. A. (1994). The evidential foundations of probabilistic reasoning. New York: J. Wiley.

Stiber, N. A., Small, M. J., \& Pantazidou, M. (2004). Site-specific updating and aggregation of Bayesian belief network models for multiple experts. Risk Analysis, 24(6), 1529-1538.

Sticha, P., Buede, D., \& Rees, R. L. (2005). APOLLO: An analytical tool for predicting a subject's decision making. Presented at the International Conference on Intelligence Analysis Methods and Tools, McLean, VA.

Surowiecki, J. (2005). The wisdom of crowds. New York, NY: Double Day.

Tetlock, P. E., \& Gardner. D. (2015). Superforecasting: The art and science of prediction., New York, NY: Crown Publishers.

Tsai, J. \& Kirlik, A. (2012). Coherence and correspondence competence: Implications for elicitation and aggregation of probabilistic forecasts of world events. Conference Proceedings of Human Factors and Ergonomics Society $56^{\text {th }}$ Annual Meeting, 313-317. Thousand Oaks, CA: Sage.

Tversky, A., \& Koehler, D. J. (1994). Support theory: A nonextensional representation of subjective probability. Psychol. Rev. 101, 547-567.

Valtorta, M., Dang, J., Goradia, H., Huang, J., \& Huhns, M. (2005). Extending Heuer's analysis of competing hypotheses method to support complex decision analysis. Presented at the International Conference on Intelligence Analysis, Washington DC.

van Gelder, T. (2008). Can we do better than ACH? Australian Institute of Professional Intelligence Officers News, 55. 
Van der Fels-Klerx, H. J., Cooke, R. M., Nauta, M. N., Goossens, L. H. \& Havelaar, A. H. (2005). A structured expert judgment study for a model of Campylobacter transmission during broiler-chicken processing. Risk Analysis, 25(1), 109-124.

Wang, G., Kulkarni, S. R., Poor, V. H., \& Osherson, D. N. (2011). Aggregating large sets of probabilistic forecasts by weighted coherent adjustment. Decision Analysis, 8(2), 128144.

Whitesmith, M. (2019). The efficacy of ACH in mitigating serial position effects and confirmation bias in an intelligence analysis scenario. Intelligence and National Security, 34(2), 225-242.

Willmott, C. J., \& Matsuura, K. (2005). Advantages of the mean absolute error (MAE) over the root mean square error (RMSE) in assessing average model performance. Climate Research, 30(1), 79-82.

Winkler, R. L., \& Clemen, R. T. (2004). Multiple experts vs. multiple methods: Combining correlation assessments. Decision Analysis, 1(3), 167-176.

Wright, G., Rowe, G., Bolger, F., \& Gammack, J. (1994). Coherence, calibration, and expertise in judgmental probability forecasting. Organizational Behavior and Human Decision Processes, 57(1), 1-25.

Zlotnick, J. (1972). Bayes' theorem for intelligence analysis. Studies in Intelligence, 16(2). Accessed April 4, 2019 at: https://www.cia.gov/library/center-for-the-study-ofintelligence/kent-csi/vol16no2/pdf/v16i2a03p.pdf 
Table I. Proportion of ACH Response Types by Presentation Condition

\begin{tabular}{lccccc}
\multirow{2}{*}{ Code } & \multicolumn{2}{c}{ Salience } & & & \\
\cline { 2 - 3 } & Low $(n=20)$ & $\operatorname{High}(n=21)$ & $\chi^{2}$ & $p$ & $\phi$ \\
\hline Rational & $0.20[0.06,0.44]$ & $.05[0.00,0.24]$ & 2.22 & .136 & 0.23 \\
Double counting & $0.25[0.09,0.49]$ & $0.57[0.34,0.78]$ & 4.36 & .037 & 0.33 \\
Other-irrational & $0.55[0.32,0.77]$ & $0.38[0.18,0.62]$ & 1.17 & .278 & 0.17 \\
\hline
\end{tabular}

Note. Values in brackets are $95 \%$ confidence intervals. 
Table II. Evaluation Metrics Comparing Warm-up and Pre-method Tasks

\begin{tabular}{|c|c|c|c|c|c|c|c|}
\hline \multirow[b]{3}{*}{ Metric } & \multicolumn{4}{|c|}{ Question Set } & \multirow[b]{3}{*}{$t$} & \multirow[b]{3}{*}{$p$} & \multirow[b]{3}{*}{$d$} \\
\hline & \multicolumn{2}{|c|}{ Warm-up } & \multicolumn{2}{|c|}{ Pre-method } & & & \\
\hline & $M$ & $S D$ & $M$ & $S D$ & & & \\
\hline $\mathrm{IM}^{\mathrm{U}}$ & 0.20 & 0.27 & 0.34 & 0.25 & 6.82 & $<.0001$ & 0.57 \\
\hline $\mathrm{IM}^{\mathrm{UA}}$ & 0.27 & 0.28 & 0.53 & 0.31 & 8.59 & $<.0001$ & 0.71 \\
\hline MAE & 0.14 & 0.15 & 0.24 & 0.12 & 9.06 & $<.0001$ & 0.75 \\
\hline
\end{tabular}


Table III. Bias from Unitarity for Pre-method and Post-method Direct Judgments

\begin{tabular}{cccccccc} 
& \multicolumn{9}{c}{ Timing } & & \multicolumn{2}{c}{} \\
\cline { 2 - 5 } Method & $M$ & $S D$ & $M$ & & & & \\
\cline { 2 - 5 } & \multicolumn{2}{c}{ Pre-method } & \multicolumn{2}{c}{ Post-method } & & $p$ & $d$ \\
\hline ACH $(n=66)$ & -0.05 & 0.71 & 0.35 & 0.56 & 5.70 & $<.0001$ & 0.70 \\
FBT $(n=81)$ & 0.16 & 0.75 & 0.09 & 0.70 & 1.03 & .30 & 0.12 \\
\hline
\end{tabular}

Note. The inferential tests show simple effects of timing, clarifying a significant method $x$ timing interaction effect. 
Table IV. Bias from Additivity for Pre-method and Post-method Direct Judgments

\begin{tabular}{|c|c|c|c|c|c|c|c|}
\hline \multirow[b]{3}{*}{ Method } & \multicolumn{4}{|c|}{ Timing } & \multirow[b]{3}{*}{$t$} & \multirow[b]{3}{*}{$p$} & \multirow[b]{3}{*}{$d$} \\
\hline & \multicolumn{2}{|c|}{ Pre-method } & \multicolumn{2}{|c|}{ Post-method } & & & \\
\hline & $M$ & $S D$ & $M$ & $S D$ & & & \\
\hline $\mathrm{ACH}(n=132)$ & 0.24 & 0.38 & 0.38 & 0.39 & 3.29 & .0013 & 0.29 \\
\hline $\operatorname{FBT}(n=162)$ & 0.28 & 0.39 & 0.29 & 0.34 & 0.45 & .65 & 0.04 \\
\hline
\end{tabular}


Table V. Accuracy by Coherentization and Timing

\begin{tabular}{lllll} 
& \multicolumn{2}{c}{ Pre-method } & \multicolumn{2}{c}{ Post-method } \\
\cline { 2 - 5 } Coherentization & $M A E$ & $S D$ & $M A E$ & $S D$ \\
\hline Raw & $0.24^{\mathrm{a}}$ & 0.12 & $0.23^{\mathrm{a}}$ & 0.11 \\
U-form & $0.12^{\mathrm{b}}$ & 0.06 & $0.13^{\mathrm{b}}$ & 0.07 \\
UA-form & $0.12^{\mathrm{b}}$ & 0.06 & $0.11^{\mathrm{c}}$ & 0.06 \\
\hline
\end{tabular}

Note. Different subscripts within columns denote pairwise differences that are significant at $p<$ .001 , whereas subscripts that do not differ are not significantly different, $p>.05$. 

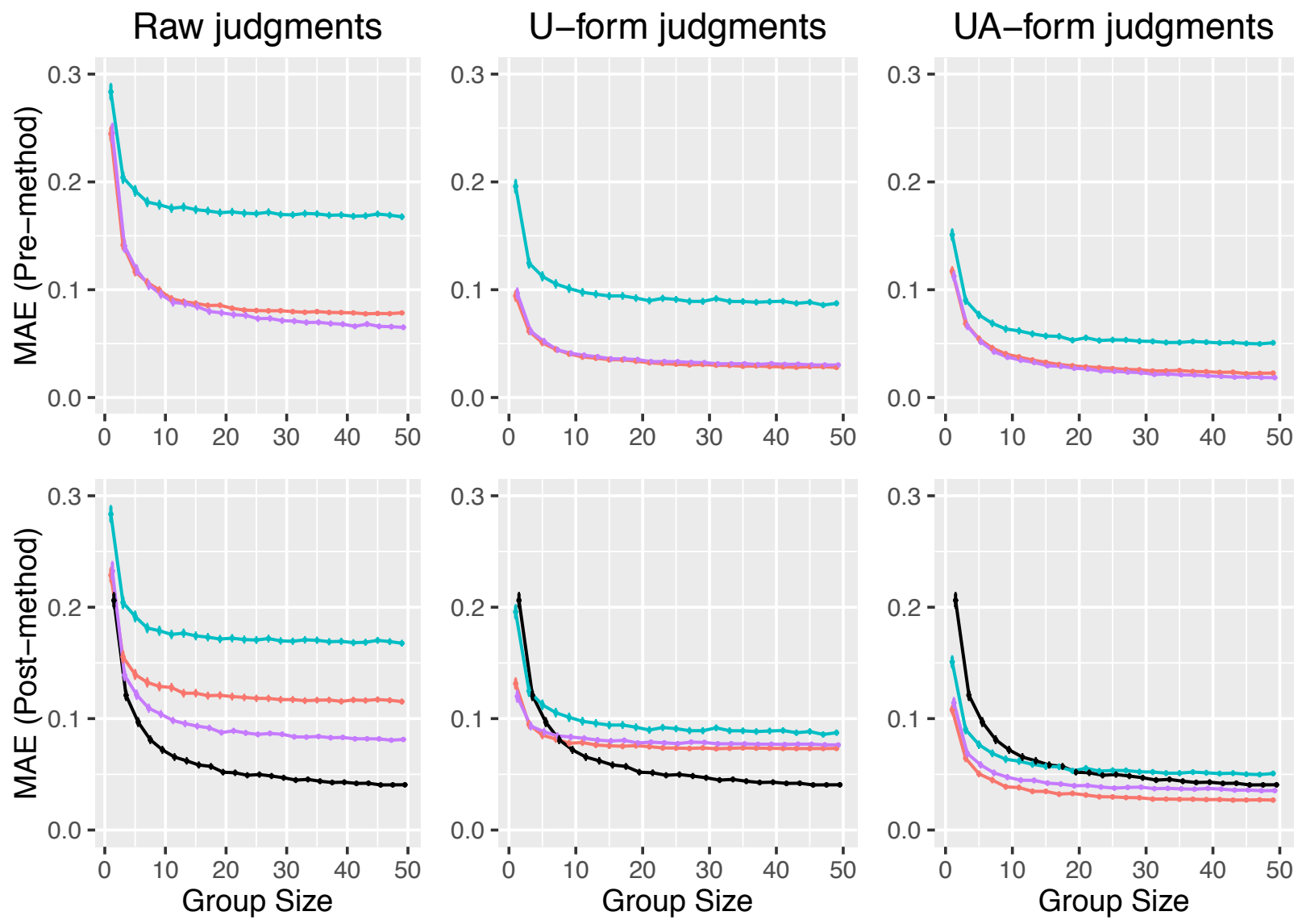

-ACH direct-FBT Computed-FBT direct--Random direct

Fig. 1. Accuracy of Unweighted Aggregation by Group Size, Analytic Method, Coherentization, and Timing 


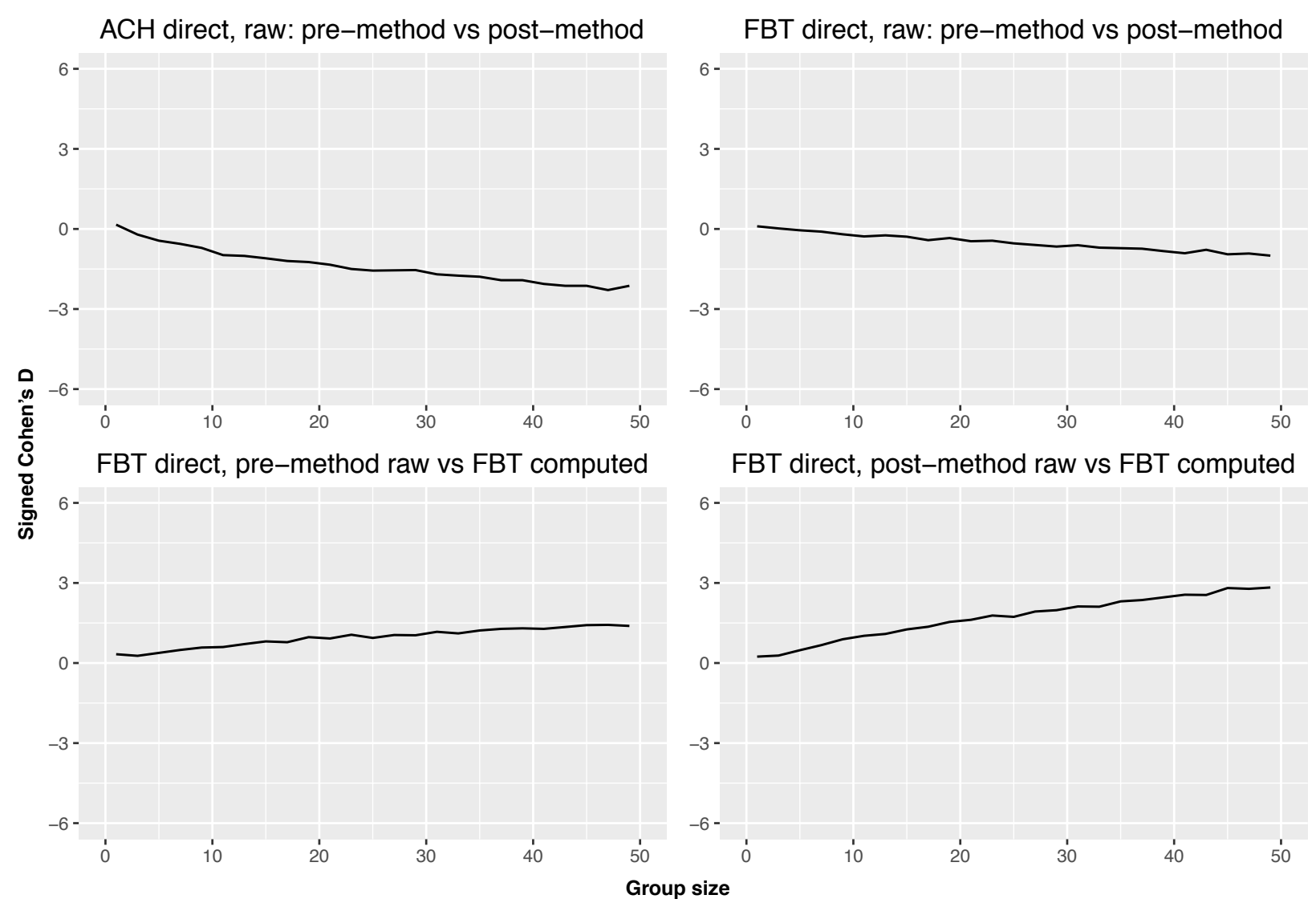

Fig. 2. Effect Sizes for Selected Pairwise Comparisons by Group Size. 

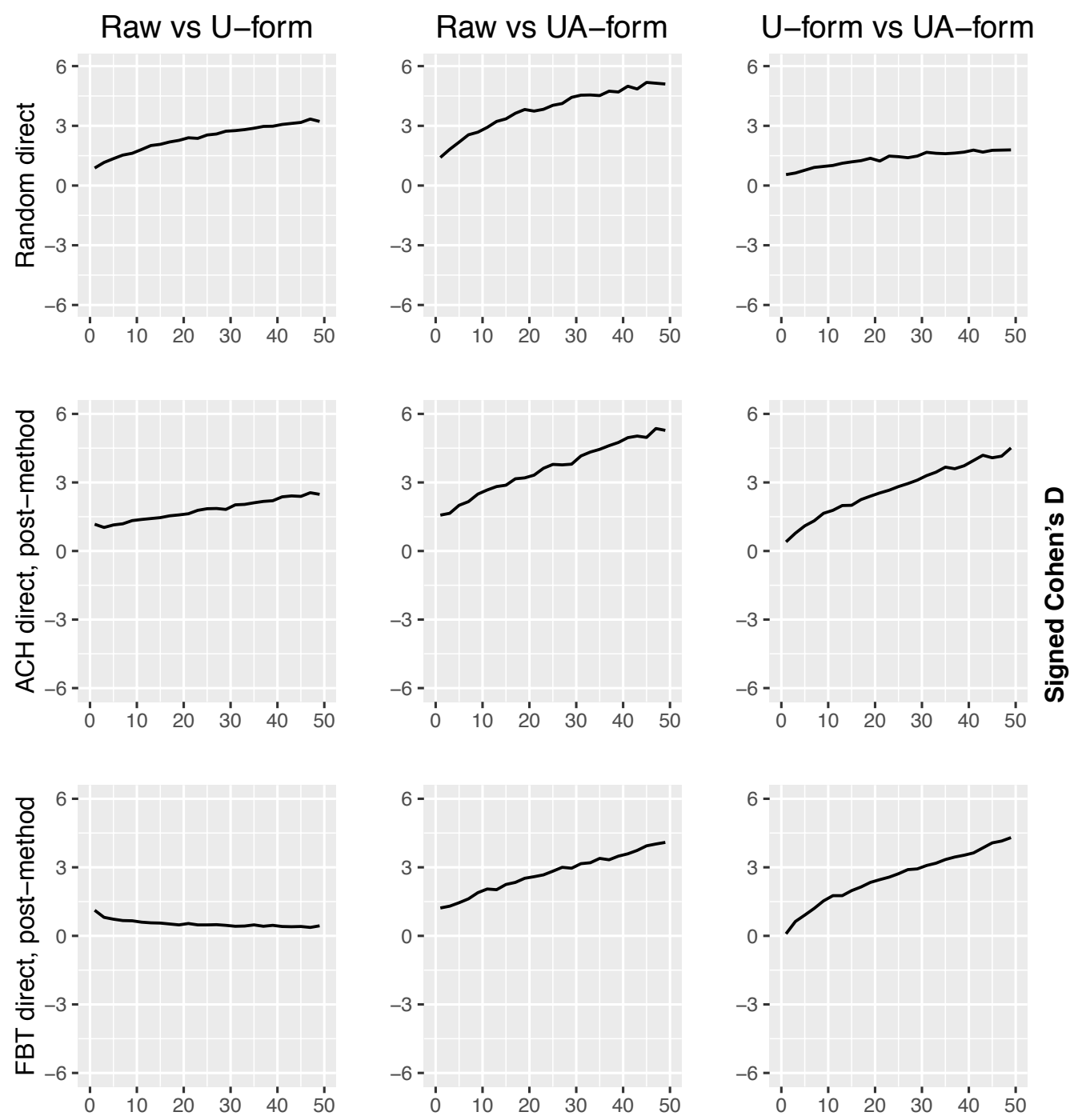

\section{Group size}

Fig. 3. Effect Sizes for Pairwise Comparisons of Coherentization Scheme by Group Size and Method. 
$\mathrm{ACH}$
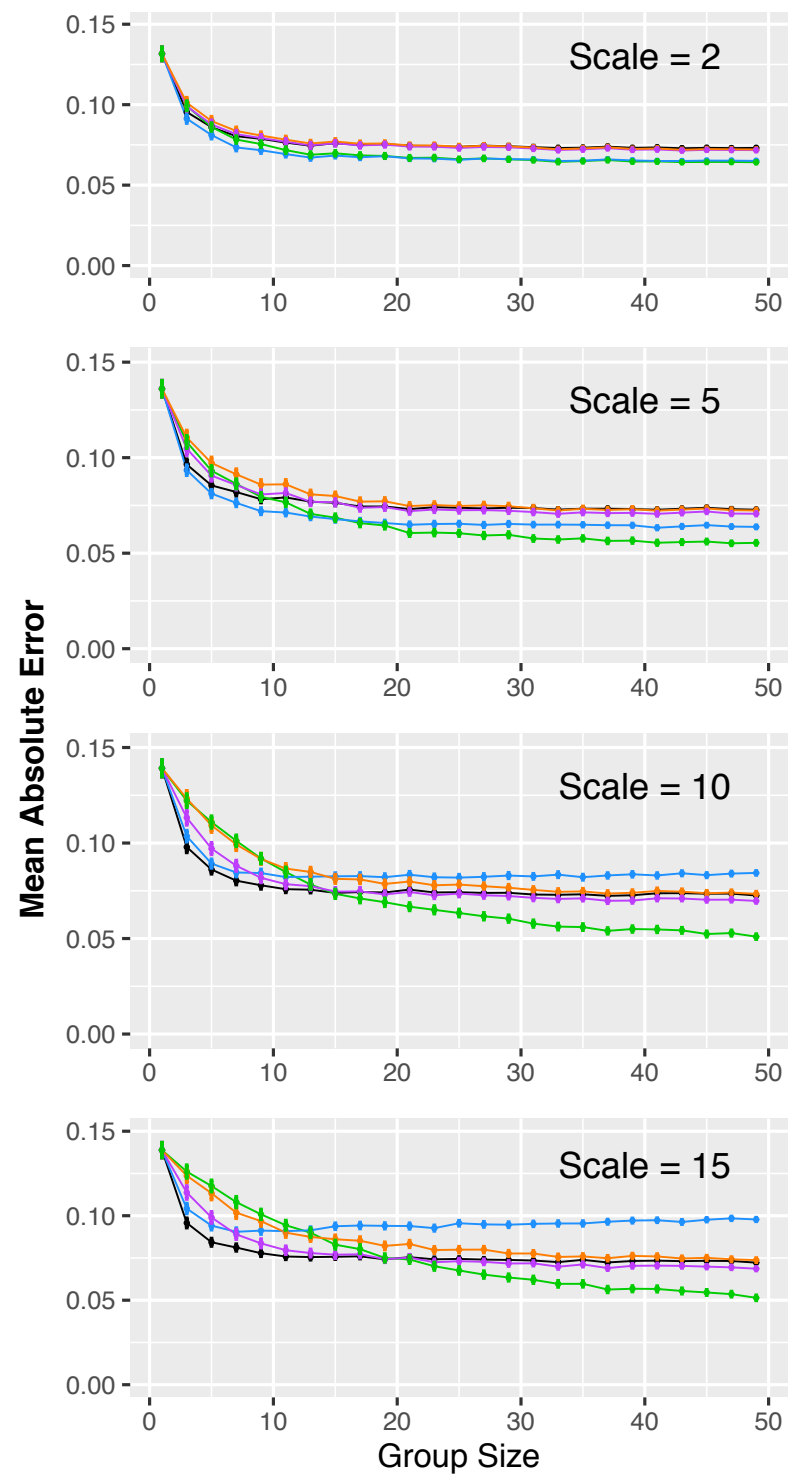

FBT
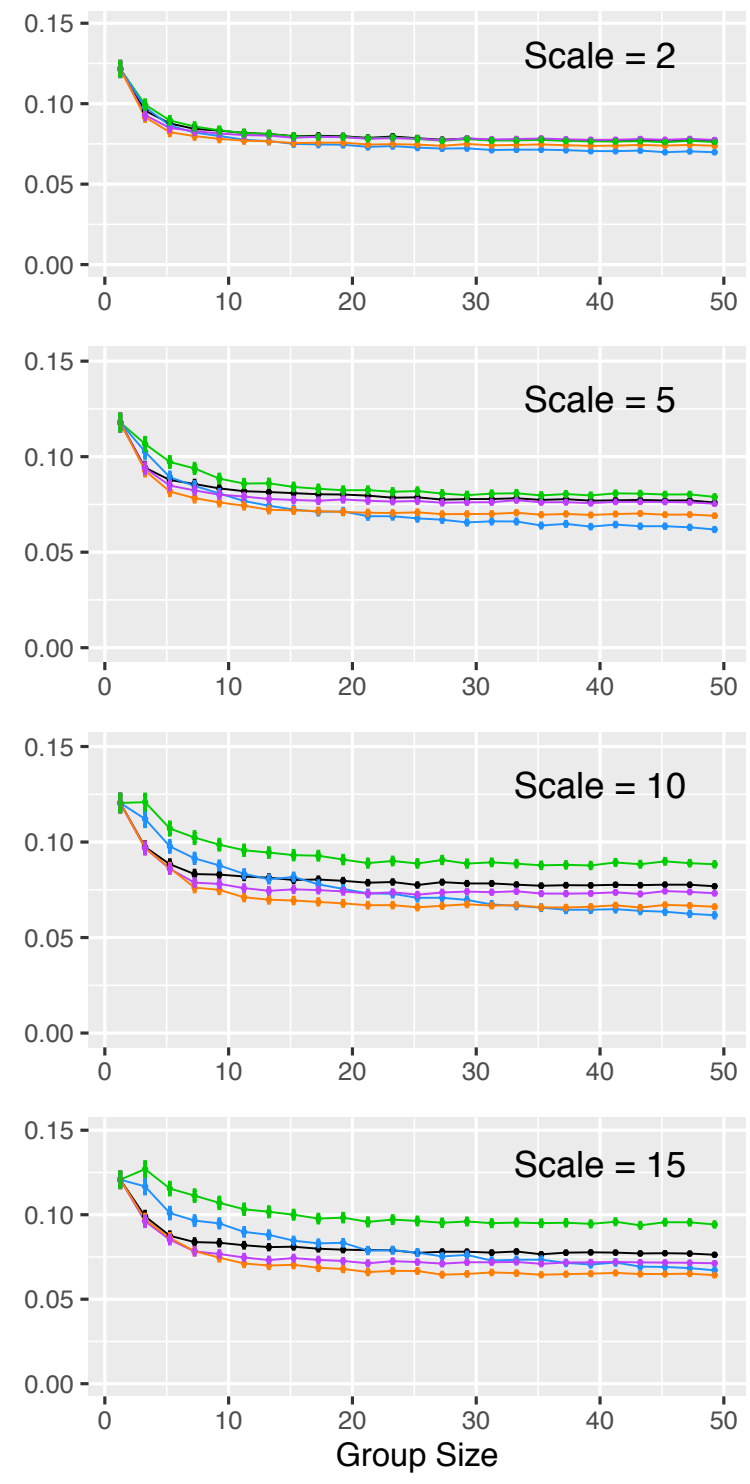

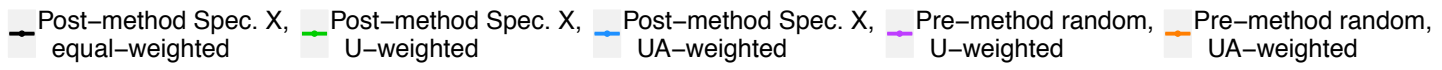

Fig. 4. Accuracy of U-form Judgments by Method, Weighting Parameter, and Weighting Method. 
$\mathrm{ACH}$
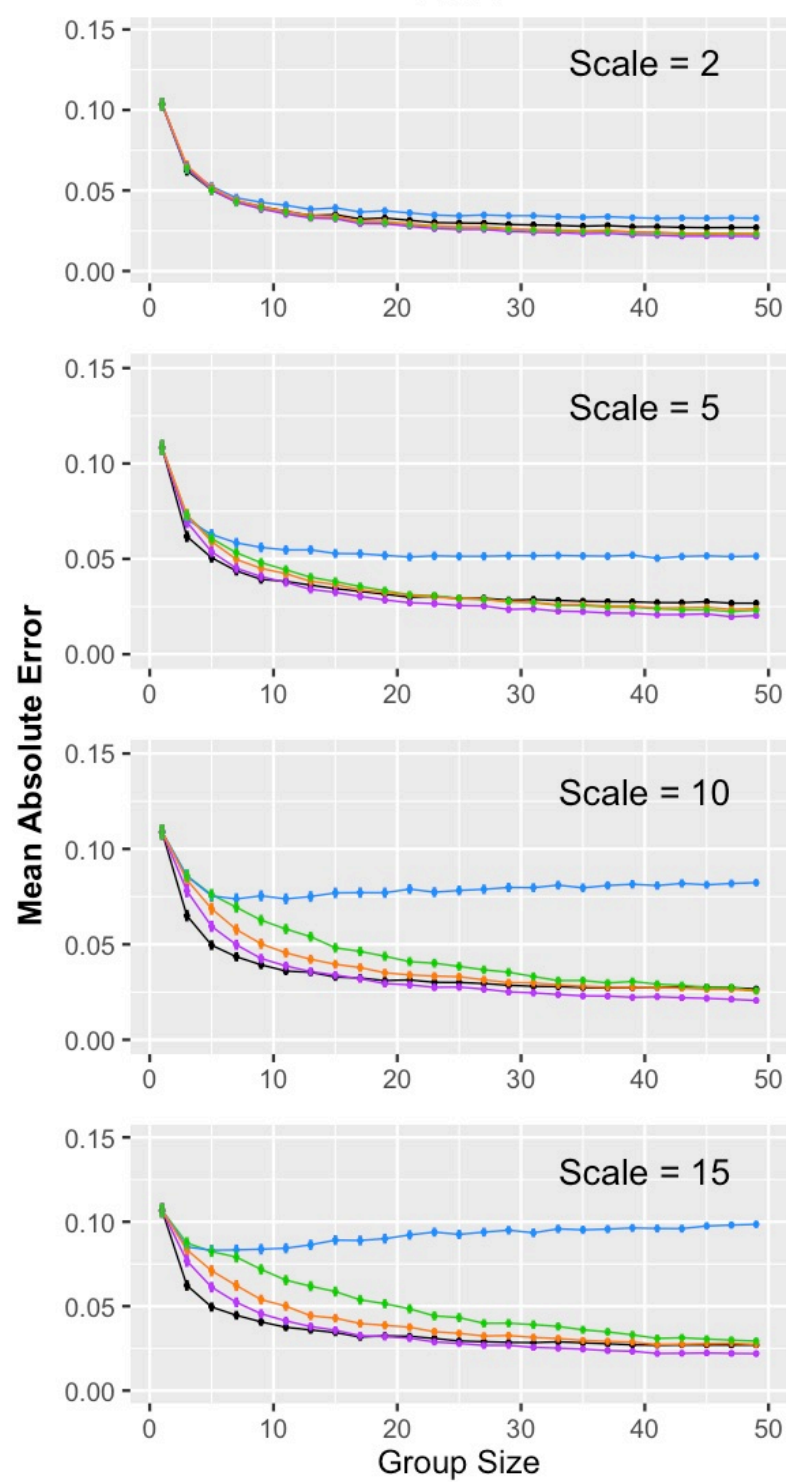
_ _ Post-method Spec. X, _ Post-method Sp UA-weighted

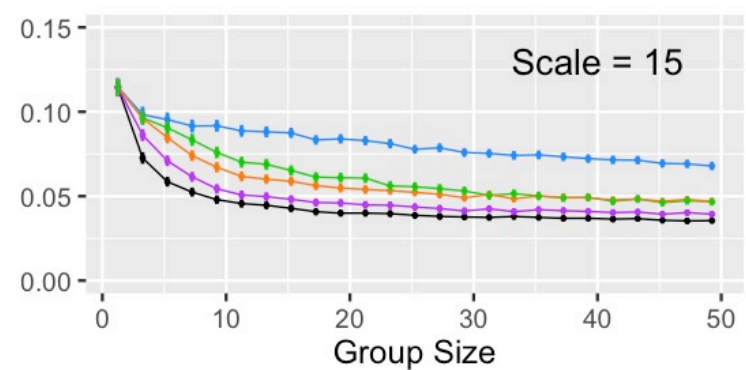

Fig. 5. Accuracy of UA-form Judgments by Group Size, Method, Weighting Parameter, and Weighting Method.
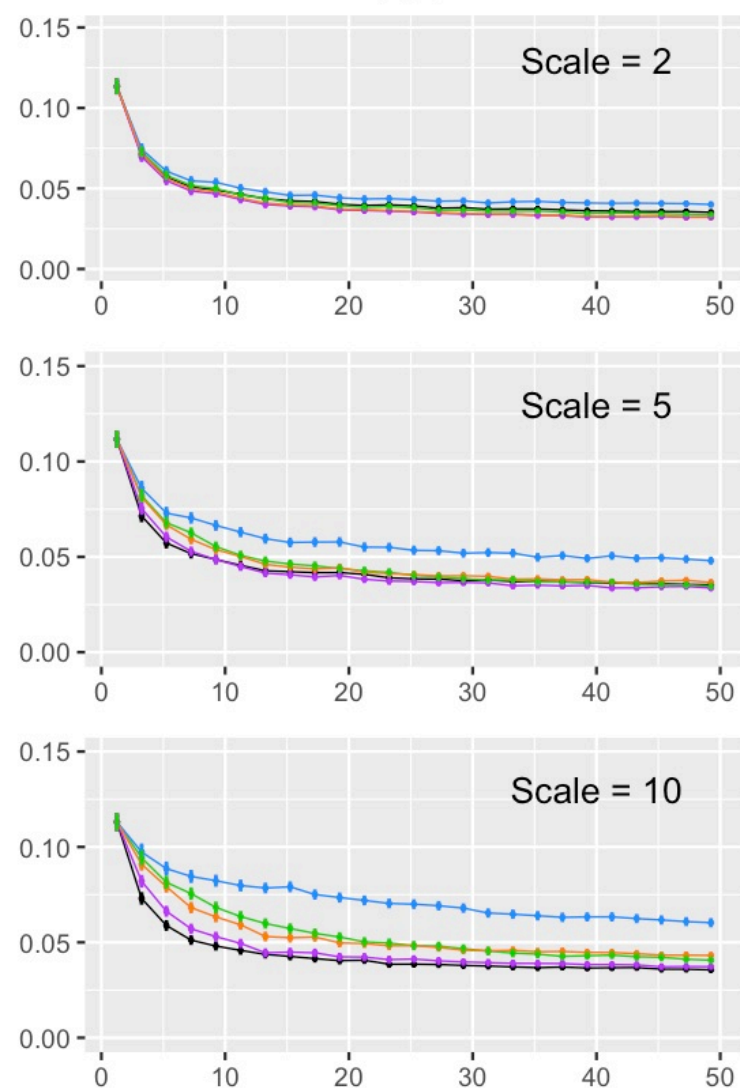


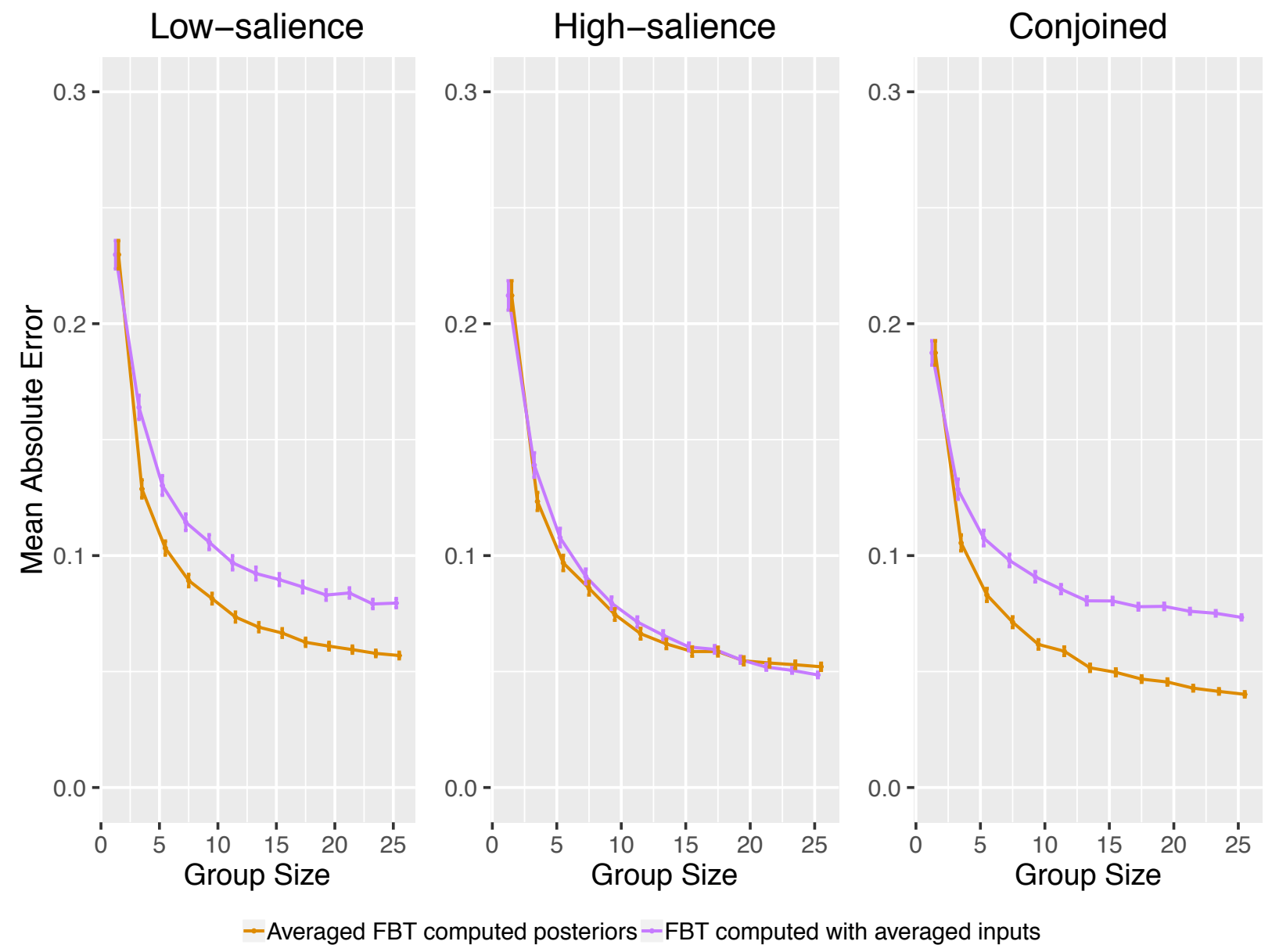

Fig. 6. Accuracy by Group Size, Presentation, and Averaging Method (Inputs vs. Outputs) in the FBT Condition. 